\title{
El empowerment como predictor del compromiso organizacional en las Pymes
}

\author{
Martha Ríos Manríquez \\ Profesora investigadora, Ins- \\ tituto Tecnológico de Celaya \\ mrm@itc.mx
}

\section{María del Rayo Téllez.}

Ramírez

Instituto Tecnológico de Celaya. terr79@hotmail.com

\section{Julián Ferrer Guerra} Profesor investigador, Instituto Tecnológico de Celaya jferrer@itc.mx

\section{Resumen}

La relación entre las variables de empowerment y compromiso organizacional ha sido un campo poco explorado desde la perspectiva organizacional. Esta investigación establece el nivel de influencia del empowerment que experimentan los colaboradores con respecto al compromiso hacia la organización para la cual laboran. Se utiliza como referencia un modelo generado con base en la descripción del empowerment en cuatro dimensiones: significado, competencia, autodeterminación e impacto; y del compromiso organizacional en tres: compromiso continuo, normativo y afectivo. Para validar el modelo propuesto, a partir de los resultados obtenidos en otros países, se administró una encuesta a 147 participantes de seis Pymes localizadas en el municipio de Celaya, Guanajuato, México. Las empresas pertenecen a los sectores de servicios, comercio y producción. Los resultados confirman las hipótesis planteadas en el sentido de que el empowerment es un predictor del compromiso organizacional; sin embargo, al analizar el impacto individual de cada una de las dimensiones se observa que sólo el compromiso afectivo tiene un impacto directo en las cuatro dimensiones planteadas.

Palabras clave: compromiso organizacional, Pymes, cultura organizacional, cambio organizacional, administración del capital humano. 


\title{
Empowerment as a predictive indicator of organizational commitment in SMEs
}

\begin{abstract}
The relationship between empowerment and organizational commitment variables has been insufficiently explored from the organizational perspective. This research analyzes the relationship between both variables in a small and medium enterprises context and sets the level of influence of empowerment on employees' commitment towards the organization they work for. We use the four dimensions of empowerment (significance, competence, self-determination and impact) in relation with three dimensions (continual, normative and affective) in organizational commitment. The model is validated with a survey of 147 employees from six Mexican SMEs in the industrial, commerce and service sectors. Outcomes confirm that empowerment can predict organizational commitment, but the individual analysis of each dimension shows that only affective commitment has a direct impact on the four dimensions of empowerment.
\end{abstract}

Keywords: organizational commitment, SME, organizational culture, organizational change, human resources management.

\section{Introducción}

Las pequeñas y medianas empresas ven limitado el crecimiento de sus operaciones debido a que realizan sus funciones de manera poco innovadora; esto se debe a una cultura de trabajo en la que los colaboradores efectúan sus actividades como las han desarrollado con anterioridad sin analizar si es la mejor forma para hacerlas. Así, los colaboradores manifiestan un escaso compromiso con la organización para la cual laboran, realizan sus funciones con un conformismo a cambio de una retribución; además, no participan ni se involucran en los procesos de operación para sugerir cambios en las decisiones, en los procesos y en los resultados, que contribuyan a mejorar a la organización.

En las organizaciones, cuyos integrantes tienen altos niveles de compromiso, se registran altos niveles de desempeño, productividad y bajos índices de ausentismo (Mowday y Steers 1979). En este sentido, pero desde otra perspectiva, existe una sensación de carencia hacia el desempeño exitoso por parte de los empleados hacia sus labores o al hacer contribuciones significativas; por ello, se requiere potenciar su actividad para tratar de eliminar la sensación de frustración y de bajos niveles 
de eficacia personal. Este estudio busca conocer el grado de influencia que ejerce el empowerment en los colaboradores para obtener un alto compromiso organizacional hacia la organización.

Actualmente, en México no existen estudios sobre el compromiso organizacional desde la perspectiva de empowerment en los colaboradores, lo que determina el nivel de la relación existente entre dichas variables. En la literatura hay pocos estudios sobre el tema, lo que indica la falta de interés de los investigadores en el intento de vincular ambas variables y, por ende, la carencia de conocimiento de los empresarios en estos temas. Lo anterior resulta paradójico si se realiza un análisis por separado de cada uno de estos temas, donde se gesta un gran conocimiento y experiencias de investigadores. En el nivel internacional, diversos autores establecen la relación entre empowerment y compromiso (Chen, 2008; Krishna, 2006; Bogler y Somech, 2004; Janssen, 2004; Dee, Henkin y Duemer 2003); mientras que Kuokkanen, Leino-Kilpi y Katajisto (2003) establecen dicha relación, pero de manera inversa, pues señalan que el compromiso determina al empowerment.

Desde la perspectiva de este trabajo, se sostiene la idea de que existe una clara relación entre ambos conceptos, especialmente se puede establecer que el empowerment determina de manera directa al compromiso organizacional. Esto es, si la organización tiene destreza para potenciar al recurso humano fomentando la autosuficiencia en conocimientos, capacidades y habilidades, entonces, dicha organización podrá alcanzar el compromiso requerido en las personas. Para algunos esta posibilidad podría implicar el rompimiento del individuo con la organización, dado que entonces la persona puede ser independiente y buscar otras posibilidades fuera de la misma. Por esto, se sostiene que no tiene que ser de esta forma, al contrario, el individuo podrá reconocer que a través de la organización logra esta posibilidad y, por lo tanto, responderá hacia la misma adquiriendo un compromiso que fortalecerá la relación entre ambos.

\section{Marco conceptual}

\section{Empowerment}

La literatura especializada ha modificado la conceptualización de los trabajadores, se refiere a ellos como capital humano y no como recurso humano. En la práctica empresarial es común encontrar la identificación del trabajador como "colaborador". Las descripciones colaborador y capital humano llevan explícitos dos com- 
promisos: primero, creer firmemente que el trabajador no es un engrane más de la maquinaria y de los sistemas de la empresa, sino que tiene el potencial suficiente para tomar decisiones y poder participar en la organización con base en sus propias capacidades; y, segundo,, existe una necesidad de desarrollar ese potencial del colaborador con educación, formación, especialización en la toma de conciencia de su papel como actor indispensable en el fortalecimiento y crecimiento de la organización.

Molina (2006) señala que el término empowerment significa habilitar, conceder, permitir una iniciativa a cierta persona para actuar por sí misma y propiciar la iniciativa en otros. Se entiende que el empowerment es sinónimo de cultura de participación que requiere del concurso de todos para alcanzar objetivos de calidad donde las organizaciones quebranten esquemas y paradigmas en cuanto a decisiones, estilos de liderazgo, equilibrio en la utilidad-beneficio dentro del eje patróntrabajador, planeación de vida y carrera del personal y, sobre todo, en los procesos de comunicación e integración de grupos humanos de trabajo en torno a los valores y la cultura organizacional. Otros aspectos que se consideran en las definiciones de dicho concepto incluyen ofrecer mayor autonomía a los empleados al compartir con ellos información relevante y darles control sobre los factores que influyen en su desempeño laboral (Davis y Newstrom, 2000); asimismo, establecerlo como un proceso estratégico que busca una relación de socios entre la organización y su gente, aumentar la confianza, responsabilidad, autoridad y compromiso para servir mejor al cliente (Jaffe y Scott, 2007).

Thomas y Velthouse (en Spreitzer, 1995) argumentan que no puede ser atribuido al colaborador únicamente, por lo que consideran cuatro dimensiones que proyectan la orientación del colaborador en su rol de trabajo: significado, competencia, autodeterminación e impacto, las cuales a continuación se describen.

- Significado. Valor que se le asigna a las metas o propósitos de trabajo en relación con los ideales o criterios propios del colaborador, involucra un conflicto entre los requerimientos del rol de trabajo y sus creencias, valores y comportamiento.

- Competencia. También llamada autoeficacia, es una creencia individual en las capacidades para desarrollar determinadas actividades haciendo uso de las habilidades que posee. Se etiqueta como competencia en lugar de autoestima porque el término se enfoca en una eficacia específica dentro del rol de trabajo, y no en la eficacia en general de un individuo. 
- Autodeterminación. Puede decirse que es el sentimiento individual de poder elegir si se inicia o no una actividad. Refleja la autonomía para iniciar y continuar un proceso o actividad en el trabajo; un ejemplo de ello es tomar la decisión sobre los métodos de trabajo, el ritmo y el esfuerzo para realizar las actividades.

- Impacto. Es el grado con el cual un colaborador puede influir estratégica, administrativa u operativamente fuera del trabajo. Cabe aclarar que la dimensión de impacto es diferente al locus de control debido que el impacto se ve influido por el contexto de trabajo, mientras que el locus de control es una característica de la personalidad que perdura a través de diversas situaciones.

Cada una de las dimensiones del empowerment en el colaborador ha sido analizada con base en los resultados que produce en la conducta y en la efectividad administrativa. El significado desarrolla una alta concentración de energía. La competencia desarrolla el esfuerzo y persistencia ante situaciones de cambio, superando las expectativas plasmadas en las metas organizacionales. La autodeterminación desarrolla el interés en actividades y la flexibilidad para enfrentar la adversidad o incertidumbre. El impacto está asociado con la ausencia para evadir situaciones difíciles, lo que genera a la vez un alto desempeño.

Esta clasificación propone de una manera completa e integral los aspectos por considerar para evaluar el empowerment en los colaboradores, se incluyen tanto los aspectos internos referentes al colaborador, como los aspectos externos a él referentes al entorno. La utilización del instrumento de evaluación del empowerment servirá para este estudio para considerar no sólo el aspecto intrínseco del colaborador, sino también el aspecto que queda fuera de su control que, de igual manera, afecta en los resultados.

\section{Compromiso organizacional}

El compromiso organizacional o lealtad de los colaboradores es definido por Davis y Newstrom (2000) como el grado en el que un colaborador se identifica con la organización y desea seguir participando activamente en ella. Los autores sostienen que el compromiso es habitualmente más fuerte entre los colaboradores con más años de servicio en una organización porque han experimentado éxito personal en la empresa, y quienes trabajan en un grupo de colaboradores comprometidos. El compromiso organizacional es un conjunto de vínculos que 
mantienen a un sujeto apegado a una organización en particular, tal como lo denomina Arciniega (2002).

Existen factores que influyen en el compromiso del colaborador, ya sea de una manera positiva o negativa y que a su vez hace referencia a un tipo de compromiso parcial - como una unidad estructural - dentro del cual un profesor puede identificarse con su departamento y no con los órganos de dirección, ni con el titular de la entidad; por ejemplo, en este contexto Gómez (2006) considera algunos factores y clasifica su contribución al compromiso organizacional de la siguiente manera: competencia personal percibida (positiva), consideración del líder (positiva), liderazgo (positiva), ambigüedad del rol (negativa) y conflictividad del puesto de trabajo (negativa).

En este mismo sentido, es esencial que la organización induzca en los colaboradores el compromiso organizacional y, al mismo tiempo, al ser dependiente de otros factores repercute en el denominado "sentimiento de pertenencia" tanto del colaborador a la organización, como de la organización al colaborador. Gómez (2006) identifica la existencia de otros factores con los que el compromiso organizacional mantiene una correlación más alta, éstos son los siguientes: motivación interna (positiva), implicación en el puesto de trabajo (positiva), satisfacción laboral global (positiva), satisfacción con el propio trabajo (positiva), satisfacción con la promoción (positiva), satisfacción con el sistema de supervisión (positiva) y estrés (negativa).

Meyer y Allen (1997) manejan tres tipos de dimensiones para el compromiso organizacional; esto no significa que existan tres variables distintas, en realidad es una sola pero en tres facetas. Las tres dimensiones del compromiso organizacional son:

- Compromiso afectivo: refleja el apego emocional, la identificación e implicación con la organización.

- Compromiso continuo: revela el apego de carácter material que el colaborador tiene con la organización, reconoce los costos asociados con dejar la organización.

- Compromiso normativo: consiste en la experimentación por parte del colaborador de un fuerte sentimiento de obligación de permanecer en la organización para la que labora, revela los sentimientos de obligación del colaborador. 
Hasta el momento se han adoptado tres perspectivas diferentes del término compromiso organizacional que se relacionan directamente con cada dimensión de compromiso antes mencionadas.

La primera es la perspectiva psicológica (compromiso afectivo) que considera el compromiso con la organización como un componente de estos tres elementos: la identificación con objetivos y valores de la organización; el deseo de contribuir para que la organización alcance sus metas y objetivos; y el deseo de ser parte de la organización. Dentro de esta perspectiva el compromiso se experimenta en relación con la organización de la cual es parte generalmente; dicha identificación y afinidad con la organización se manifiesta a través de actitudes como el orgullo de permanecer en la organización cuando se emociona al mencionar que trabaja en ella, o cuando habla mucho de la organización en reuniones de familiares o amigos, también se refleja en la solidaridad que muestra el colaborador al sentirse preocupado cuando la organización está pasando por una mala racha, o cuando se alegra de que las cosas funcionen bien dentro de la organización.

La segunda es la perspectiva de intercambio (compromiso continuo) que sugiere que el compromiso organizacional es el resultado de una transacción de incentivos y contribuciones entre la organización y el colaborador. De acuerdo con este concepto, el colaborador asocia los beneficios que recibe con el trabajo, tales como plan de retiro y seguro de salud y los considera como incentivos para permanecer en la organización (Bayona, Goñi y Madorrán 1999). Otro aspecto que toma en cuenta el colaborador son las oportunidades que tiene para conseguir un nuevo trabajo en condiciones similares. De esta manera, si el trabajador observa que tiene mayores posibilidades, entonces será menor su apego a la organización; por el contrario, mientras más reducidas sean las posibilidades, el resultado será un mayor apego a la organización. Las variables como la edad, antigüedad en el trabajo, nivel educativo y la capacitación recibida influyen directamente en el compromiso continuo. Cabe mencionar que a mayor edad y antigüedad en la organización, mayor será su compromiso con ésta; en cambio, mientras mayor sea el nivel educativo y la capacitación recibida por parte de un colaborador, menor será su compromiso ya que se amplían sus posibilidades de aportar sus conocimientos a otra organización. Podemos asumir que cuando un colaborador tiene un compromiso continuo fuerte, pero su compromiso afectivo y normativo es menor, su esfuerzo, dedicación y entrega en el trabajo sólo será el mínimo para permanecer en la organización, y esperar a tener mejores oportunidades externas para dejar a la organización. 
La tercera es la perspectiva de atribución (compromiso normativo) que define el compromiso como una obligación que el colaborador adquiere como resultado de realizar ciertos actos que son voluntarios, explícitos e irrevocables. Dentro de esta categoría se ubica el compromiso organizacional que los miembros de grupos religiosos adquieren cuando pronuncian públicamente sus votos religiosos o cuando funcionarios públicos juran cumplir con sus obligaciones en los actos de toma de posesión. Al igual que el compromiso afectivo, el compromiso normativo es de carácter emocional porque desarrolla un sentimiento de obligación hacia la organización que puede tener su origen desde la formación del colaborador en su niñez, adolescencia o sus primeras experiencias laborales. Cuando un colaborador desarrolla un alto compromiso normativo, consecuentemente puede experimentar un sentimiento de deuda hacia la organización que le brindó la oportunidad de trabajar, por lo que su trabajo puede ser incondicional al momento de implementar algún nuevo proyecto, donde tal vez dicho colaborador no trabajará con el entusiasmo y energía del colaborador con compromiso afectivo, pero sí con la seguridad y garantía de que en todo momento apoyará a la organización.

Por otra parte, los colaboradores que tienen un compromiso afectivo fuerte permanecen en la empresa porque "quieren"; los que tienen un fuerte compromiso continuo permanecen porque "lo necesitan"; y los que tienen un fuerte compromiso normativo permanecen porque sienten que "deben" hacerlo. Evaluar el compromiso organizacional desde los tres posibles sentimientos que experimenta un colaborador brinda la oportunidad de obtener resultados más completos al definir el tipo de compromiso que experimentan y predecir su posible reacción ante determinados estímulos.

\section{La relación empowerment-compromiso organizacional}

La literatura existente no presenta muchos resultados sobre estudios que analicen la relación de ambos conceptos. Sin embargo, es posible rescatar los resultados obtenidos por algunos autores en el ámbito internacional. Para proponer el uso del empowerment para fortalecer el compromiso de los colaboradores hacia la organización y examinar los efectos sobre el locus de control y la percepción del contrato psicológico bajo condiciones de cambio, Chen (2008) estableció la evidencia de influencia del mismo sobre el compromiso organizacional. Los resultados muestran que el empowerment percibido por los colaboradores que tienen un locus externo, así como quienes perciben una fuerte adhesión al contrato psicológico tienen un impacto positivo fuerte sobre el compromiso organizacional. Krishna (2006) 
observó una significativa relación positiva entre el empowerment psicológico con el compromiso afectivo y el compromiso normativo; sin embargo, la relación no fue encontrada entre la dimensión de competencia de la variable empowerment y la dimensión de compromiso afectivo de la variable de compromiso organizacional. Se concluye que los colaboradores con empowerment muestran los niveles más altos de compromiso organizacional.

Un estudio de la relación entre el empowerment, compromiso organizacional, compromiso profesional y conducta ciudadana organizacional — realizado por Bogler y Somech en 2004- mostró que el grado de empowerment se encuentra estrechamente relacionado con los sentimientos de compromiso tanto a la organización, como a su profesión y hacia la conducta ciudadana organizacional. La subdivisión de la variable de empowerment en seis subescalas muestra que el crecimiento profesional, el estatus y la autoeficacia son importantes predictores del compromiso organizacional y del profesional; mientras que la escala de toma de decisiones, la autoeficacia y el estatus son predictores significativos de la conducta ciudadana organizacional. Janssen (2004) estableció el supuesto de que existe conflicto de tipo laboral con los superiores, lo que genera una barrera en la relación positiva entre el empowerment y el compromiso organizacional. Se encontró que los superiores, quienes tienen un grado de autoridad más alto, son quienes persiguen las metas y los valores de la organización.

Dee, Henkin y Duemer (2003) en un estudio entre maestros encontraron que los facultados mostraron mayores niveles de compromiso organizacional, por lo que establecieron que el empowerment puede ser un importante medio para disminuir el nivel de hastío de los maestros y la alta rotación. Por su parte, Kuokkanen, Leino-Kilpi y Katajisto (2003) destacaron el hecho de que la satisfacción profesional y el compromiso hacia la organización son elementos cruciales para el empowerment de la enfermera.

\section{Métodos y medios}

\section{Tipo de investigación y variables}

Esta investigación se cataloga como una investigación no experimental porque se realiza el estudio de una situación ya existente en las organizaciones donde no se tiene control o influencia para relacionar las variables que se están investigando; es decir, los colaboradores ya poseían su propia percepción acerca del nivel de 
sus capacidades frente al desarrollo de su trabajo (término conocido como empowerment), también tenían un cierto grado de compromiso hacia la organización al momento de aplicar los instrumentos de evaluación. De la misma forma corresponde al tipo correlacional debido a que busca medir el grado de relación entre la variable de empowerment y la variable de compromiso organizacional. Finalmente, es establecida como causal dado que se pretende obtener evidencia de la relación causa-efecto que produce el empoderamiento de los colaboradores en la adhesión hacia la empresa. El estudio, que comprendió de diciembre 2008 a mayo 2009, se realizó en las instalaciones de empresas ubicadas en el municipio de Celaya, Guanajuato.

El objetivo del estudio consistió en explicar el comportamiento del compromiso organizacional (variable dependiente) con base en el empowerment de los colaboradores (variable dependiente). Para efectos de este proyecto ambas variables han sido operacionalizadas de la siguiente manera: empowerment es el nivel de independencia que posee un individuo para desarrollar las actividades que le son encomendadas; mientras que el compromiso organizacional se considera como el grado de adhesión que un individuo experimenta hacia la organización que labora.

\section{Figura1}

Modelo predictivo empowerment - compromiso organizacional

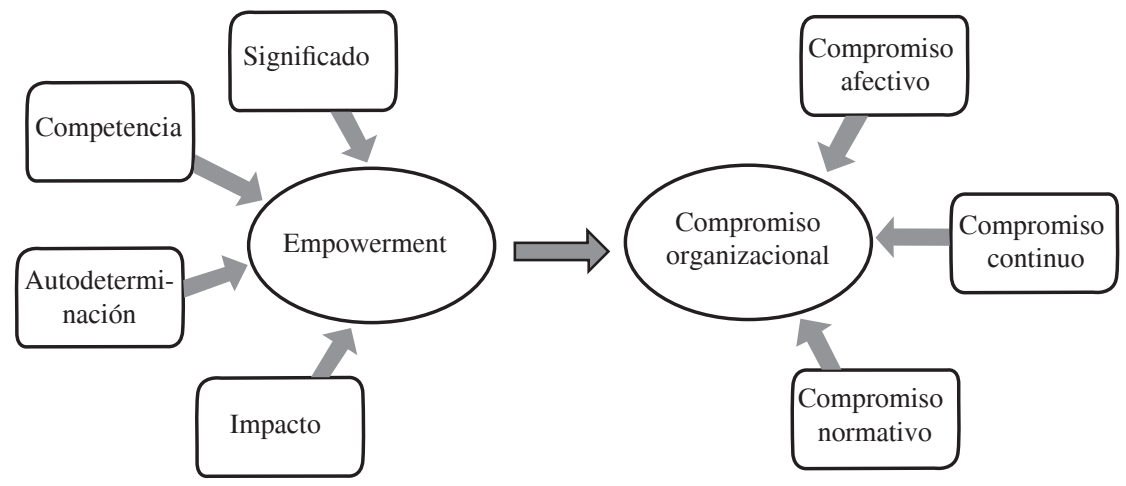

Fuente: Elaboración propia basada en Spreitzer (1995), Meyer y Allen (1997)

La figura 1 presenta el modelo por evaluar, el cual se basa en las teorías propuestas por Spreitzer (1995) — para la variable de empowerment — y de Meyer y Allen (1997). En la primera variable, donde se consideran cuatro dimensiones: significa- 
do, competencia, autodeterminación e impacto, influye directamente en la variable de compromiso organizacional, que se divide en tres dimensiones: compromiso afectivo, compromiso continuo y compromiso normativo.

\section{Hipótesis}

Hipótesis nula:

$\mathrm{H}_{0}$ : No existe relación de influencia del empowerment respecto con el compromiso organizacional.

Hipótesis alternativas:

$\mathrm{H}_{1}$ : Entre mayor empowerment se otorgue a los colaboradores, mayor será el compromiso organizacional que desarrollen.

$\mathrm{H}_{1 \mathrm{~A}}$ : El significado, competencia, autodeterminación e impacto como dimensiones del empowerment determinan de manera directa el compromiso organizacional.

$\mathrm{H}_{1 \mathrm{~B}}$ : El empowerment determina de manera directa las dimensiones de compromiso afectivo, continuo y normativo del compromiso organizacional.

Cabe señalar que se obtuvo información adicional de los factores demográficos en la población objeto de estudio, tales como la organización a la cual pertenecen, género, edad, antiguiedad en la organización, tipo de puesto que desempeñan y la escolaridad de los investigados.

\section{Descripción de la población}

El número de empresas participantes se determinó de acuerdo con el muestreo por conveniencia (Salkind 1999). Malhotra (2004) señala que de este tipo de muestreo no probabilístico se obtiene una cantidad de elementos adecuados al estudio porque es la técnica menos costosa y requiere menor tiempo con unidades de estudio más accesibles y cooperativas, pero tiene riesgos inherentes en las posibles fuentes de sesgo en la información y la limitante de no poder generalizar los resultados obtenidos. Se determinó realizar el estudio en seis empresas (tres pequeñas y tres medianas), las cuales están en los giros de comercio, servicios profesionales, industrial y de la construcción. En total se administraron 147 en- 
cuestas, en las medianas se realizó un muestreo aleatorio simple, mientras que para las pequeñas se realizó un censo.

\section{Instrumentos de recolección de datos}

Para evaluar el nivel de empowerment experimentado por los colaboradores en la organización fue utilizada la escala propuesta por Spreitzer (1995) debido a que su aplicación al colaborador se realiza en forma individual sin forzar a que las respuestas se encuentren sujetas a la evaluación del trabajo que se desarrolla en equipo con sus compañeros. Para el compromiso organizacional se seleccionó la propuesta de Meyer y Allen (1997) porque sus dimensiones permiten explicar de mejor manera los sentimientos que desarrolla el colaborador con respecto de la organización; se tomó en cuenta si el colaborador desea, se ve obligado o económicamente necesita mantener la relación laboral. Para ambos instrumentos se utilizó la escala tipo Likert de seis niveles que va de fuertemente en desacuerdo (1) a fuertemente de acuerdo (6).

Los instrumentos fueron traducidos del inglés al español, se sometieron a una prueba piloto, que incluye un análisis de contenido, en la cual participaron dos empresarios de las organizaciones objeto de estudio y un grupo de estudiantes de diferentes niveles educativos con el fin de asegurar las adecuaciones necesarias para una redacción equivalente al lenguaje comúnmente utilizado en México en diferentes niveles de educación escolar.

Para determinar la fiabilidad de los instrumentos se evaluó el alfa de Combrach para cada una de las subescalas que incluyó el cuestionario. De manera general, se puede afirmar que los instrumentos utilizados proporcionan una evaluación adecuada, como se observa en el cuadro 1. Cada una de las dimensiones obtuvo un alfa mayor de 0.70 de acuerdo con lo sugerido por Nunnally (1978), a excepción de la dimensión de competencia (empowerment) a razón de 0.573 , lo cual es consistente con el estudio original en el que se obtuvo un alfa de 0.58 . 


\section{Cuadro 1}

Resumen análisis de fiabilidad para cada dimensión (Alfa de Combrach)

\begin{tabular}{|c|c|c|c|c|c|c|c|}
\hline \multirow[t]{2}{*}{ Empowerment } & \multirow{2}{*}{$\begin{array}{l}\text { Estudio } \\
\text { actual }\end{array}$} & \multicolumn{2}{|c|}{$\begin{array}{c}\text { Spreitzer } \\
(1995: 1457)\end{array}$} & \multirow{2}{*}{$\begin{array}{c}\text { Compromiso } \\
\text { organizacional }\end{array}$} & \multirow{2}{*}{$\begin{array}{c}\text { Estudio } \\
\text { Actual }\end{array}$} & \multicolumn{2}{|c|}{$\begin{array}{c}\text { Rego y Souto } \\
\text { (2004) }\end{array}$} \\
\hline & & Industria & Aseguradoras & & & Portugal & Brasil \\
\hline Significado & 0.837 & 0.72 & 0.83 & Afectivo & 0.847 & 0.82 & 0.81 \\
\hline Competencia & 0.573 & 0.58 & 0.74 & Normativo & 0.822 & 0.83 & 0.67 \\
\hline Autodeterminación & 0.749 & 0.92 & 0.49 & Continuo & 0.905 & 0.83 & 0.80 \\
\hline Impacto & 0.763 & 0.92 & 0.49 & & & & \\
\hline
\end{tabular}

\section{Composición demográfica}

Las tres empresas pequeñas cuentan con 11, 13 y 16 empleados, respectivamente, mientras que las medianas con 55, 59 y 63 empleados. Del total de encuestados, $25 \%$ corresponden a empleados de las pequeñas empresas y $75 \%$ a empleados de medianas empresas. $71 \%$ fueron de género masculino y $29 \%$ femenino. En la edad el $88 \%$ es menor de 40 años (la mayor proporción se encuentra entre 21 y 30 años con 40\%). Con respecto a la antiguiedad de los colaboradores dentro de la organización donde prestan sus servicios, se observa que uno de cada dos empleados tiene una antigüedad menor de dos años (ver cuadro 2), lo que implica una baja permanencia laboral de los colaboradores. El 84\% de los encuestados corresponde a personal operativo.

\section{Cuadro 2}

\section{Antigüedad y tipo de función dentro de la organización}

\begin{tabular}{cccc}
\hline Antigüiedad & Porcentaje & Puesto & Porcentaje \\
\hline Hasta 2 años & $52 \%$ & Administrativo & $16 \%$ \\
De 3 a 4 años & $20 \%$ & Operativo & $84 \%$ \\
Más de 5 años & $28 \%$ & & \\
\hline Total & $100 \%$ & Total & $100 \%$
\end{tabular}

Finalmente, dentro del nivel de escolaridad de los colaboradores $37 \%$ cuenta con estudios de nivel básico, 39\% tiene escolaridad a nivel medio superior y sólo el 24\% cuenta con estudios de nivel licenciatura. Las dimensiones de la variable empowerment manifiestan niveles mayores a los reflejados por las dimensiones del compromiso organizacional, lo que índica que es necesario facultar a los colaboradores en una proporción de 1.13 a 1 (5.23/4.60), con la finalidad de obtener el grado de compromiso deseado (ver cuadro 3). De manera contraria, se observa una mayor desviación estándar en el compromiso organizacional, lo que implica una mayor dispersión de la concepción de esta variable por parte de los colaboradores. 
Cuadro 3

Estadísticos por dimensión en empowerment y compromiso organizacional

\begin{tabular}{ccc}
\hline Dimensión & Media & Desviación estándar \\
\hline Significado & 5.49 & 0.742 \\
Competencia & 5.48 & 0.729 \\
Autodeterminación & 5.14 & 1.002 \\
Impacto & 4.80 & 1.110 \\
Empowerment & 5.23 & 0.896 \\
Compromiso afectivo & 4.67 & 1.187 \\
Compromiso normativo & 4.67 & 1.232 \\
Compromiso continuo & 4.47 & 1.423 \\
Compromiso Organizacional & 4.60 & 1.281 \\
\hline
\end{tabular}

Se observó que las pequeñas empresas poseen los niveles más altos en empowerment, mientras que las medianas empresas observaron niveles superiores en compromiso organizacional, tal y como lo muestra la información del cuadro 4.

\section{Cuadro 4}

Valores obtenidos por variables moderadoras

\begin{tabular}{ccccc}
\hline & \multicolumn{2}{c}{ Empowerment } & \multicolumn{2}{c}{ Compromiso organizacional } \\
\hline & Media & Desviación Std & Media & Desviación Std \\
\hline \multicolumn{4}{c}{ Tipo de Puesto } \\
\hline Administrativo & 5.3576 & .5802 & 4.3316 & .7655 \\
Operativo & 5.2073 & .5488 & 4.6643 & .7445 \\
\hline \multicolumn{5}{c}{ Género de los colaboradores } \\
\hline Hombre & 5.2099 & 0.57089 & 4.5986 & 0.71314 \\
Mujer & 5.2849 & 0.51634 & 4.6376 & 0.85775 \\
\hline \multicolumn{5}{c}{ Edad de los colaboradores } \\
\hline Hasta 20 años & 4.9107 & 0.57598 & 4.4271 & 0.64086 \\
De 21 a 30 años & 5.2874 & 0.56738 & 4.4648 & 0.87362 \\
De 31 a 40 años & 5.2907 & 0.51253 & 4.7519 & 0.67810 \\
Más de 40 años & 5.4120 & 0.40571 & 5.0231 & 0.44240 \\
\hline & 5.4167 & Escolaridad de los colaboradores & 0.54499 \\
\hline Primaria & 0.46595 & 4.9646 & 0.50606 \\
Secundaria & 5.2353 & 0.57861 & 4.9608 & 0.68559 \\
Técnico superior & 5.2157 & 0.61946 & 4.7353 & 0.88820 \\
Preparatoria & 5.0354 & 0.52735 & 4.5115 & 0.66260 \\
Licenciatura & 5.3519 & 0.53531 & 4.1319 & \\
\hline
\end{tabular}


El tipo de organización constituye un factor importante para la toma de decisiones, se puede decir que en pequeñas empresas el nivel de empowerment en los colaboradores es mayor considerando que las funciones que desempeñan no se encuentran tan especializadas como sucede en organizaciones que crecen en tamaño, otorgándoles la seguridad en realizar actividades de diversa índole dentro de la organización; en tanto que el compromiso organizacional es mayor en medianas empresas si se toma en cuenta que generalmente son mayores los beneficios económicos y en especie que reciben los colaboradores comparados con los que se ofrecen en las pequeñas empresas.

Con respecto a la antigüedad, se observó que los colaboradores de reciente ingreso y aquellos que tienen cierta permanencia en la organización tienen mayor empowerment y compromiso hacia la empresa, mientras que los trabajadores intermedios en su antigüedad reflejan niveles inferiores. Los resultados de acuerdo con las funciones que desempeñan los colaboradores mostraron que los niveles más altos de empowerment los experimentan quienes realizan funciones de tipo administrativo; contrariamente, encontramos que los niveles más elevados en la variable de compromiso organizacional son asignados a colaboradores que cumplen con funciones operativas (ver cuadro 4); asimismo, se encontró una tendencia inversa en el nivel de empowerment y compromiso organizacional en relación con el tipo de puesto.

En la perspectiva de género, el sexo femenino tuvo las puntuaciones más altas en variables (cuadro 4), este hallazgo se explica para el compromiso organizacional al considerar las costumbres desarrolladas en la cultura organizacional expuestas por Robbins (2004), quien se basó en las dimensiones culturales de Hosftede, las cuales mostraron que en México existe una distancia de poder grande con poca incertidumbre y con una fuerte masculinidad.

Los resultados obtenidos en función de la edad mostraron que mientras menor sea ésta, será menor el nivel de emporwerment y de compromiso. Esto se modifica en la medida que la edad aumenta. Con respecto al nivel académico, se encontró que ambas variables de estudio disminuyen conforme dicho nivel se incrementa en los colaboradores, es mayor el resultado donde es menor la escolaridad. Con base en los resultados, se infiere que conforme los empleados incrementan sus conocimientos, aumenta la libertad, independencia y seguridad en sí mismos para generar la búsqueda de alternativas de trabajo en otras organizaciones que satisfagan sus requerimientos. 


\section{Análisis y discusión de resultados}

\section{Análisis de correlación}

Con el objeto de analizar el comportamiento de las diversas dimensiones de cada una de las dos variables del modelo, se elaboró un análisis de correlación bivariado de Pearson (cuadro 5).

\section{Cuadro 5}

\section{Correlaciones entre dimensiones de empowerment y compromiso organizacional}

\begin{tabular}{|c|c|c|c|c|c|c|}
\hline & Significado & Competencia & $\begin{array}{l}\text { Autodeter- } \\
\text { minación }\end{array}$ & Impacto & $\begin{array}{c}\text { Compromiso } \\
\text { afectivo }\end{array}$ & $\begin{array}{c}\text { Compromiso } \\
\text { normativo }\end{array}$ \\
\hline Significado & 1 & & & & & \\
\hline Competencia & $0.408 * *$ & 1 & & & & \\
\hline Autodeterminación & $0.337 * *$ & $0.530 * *$ & 1 & & & \\
\hline Impacto & $0.347 * *$ & $0.372 * *$ & $0.505 * *$ & 1 & & \\
\hline $\begin{array}{l}\text { Compromiso } \\
\text { afectivo }\end{array}$ & $0.555^{* *}$ & $0.248 * *$ & $0.318^{* *}$ & $0.307 * *$ & 1 & \\
\hline $\begin{array}{c}\text { Compromiso } \\
\text { normativo }\end{array}$ & $0.451 * *$ & $0.170^{*}$ & $0.199 *$ & $0.169 *$ & $0.594 * *$ & 1 \\
\hline $\begin{array}{c}\text { Compromiso } \\
\text { continuo }\end{array}$ & $0.169^{*}$ & -0.017 & 0.069 & 0.063 & $0.286^{* *}$ & $0.638 * *$ \\
\hline
\end{tabular}

** La correlación es significativa al nivel 0,01 (bilateral)

* La correlación es significante al nivel 0,05 (bilateral)

Es posible asumir el hecho de que las dimensiones de significado, competencia, autodeterminación e impacto que explican a la variable independiente de empowerment, manifiestan un vínculo de medio a fuerte entre ellas. La figura 2 presenta el modelo de investigación que refleja la interrelación de las dimensiones de las variables incluidas en dicho modelo, se encontró que los niveles de correlación más altos corresponden a la interrelación de la dimensión de autodeterminación con la de competencia y la dimensión de impacto a un nivel de 0.530 y 0.505 , respectivamente. Se observó también que la interrelación más baja se encuentra con un nivel de 0.337 entre las variables de autodeterminación y significado, lo que arroja una correlación significativa estadísticamente. 
Figura 2

Correlación empowerment - compromiso organizacional dimensiones estadísticamente significativas (al nivel de $\mathbf{0 . 0 1}$ )

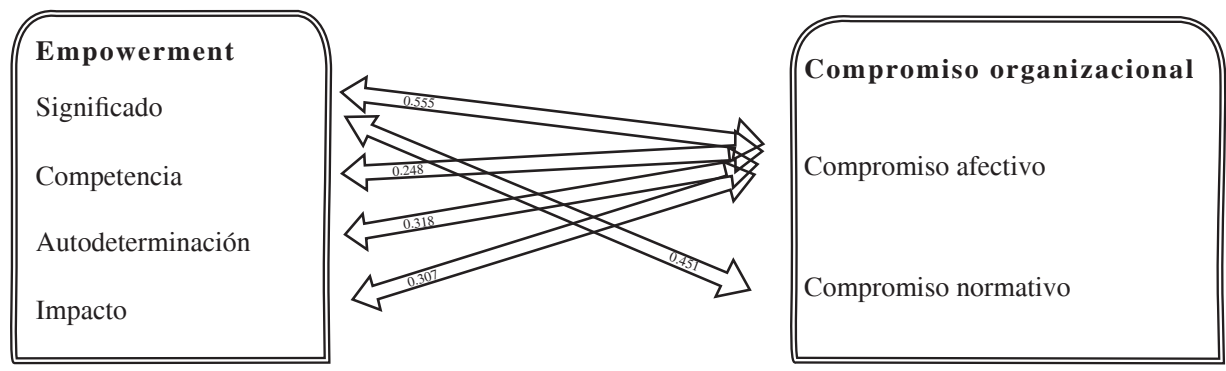

Fuente de elaboración propia

\section{Análisis de regresión}

Para predecir el comportamiento de la variable dependiente (compromiso organizacional) se utilizó el análisis de regresión simple. En dicho análisis se consideraron las dimensiones de significado, competencia, autodeterminación e impacto de la variable empowerment (cuadro 6). Se obtuvo una distribución F de 9.892, que es el valor esperado de la misma (2.49), por lo cual se acepta la hipótesis alternativa.

\section{Cuadro 6}

\section{Análisis de regresión}

\begin{tabular}{c|c|c|c|c|c}
\hline Modelo & $\begin{array}{c}\text { Suma } \\
\text { de cuadrados }\end{array}$ & $\begin{array}{c}\text { Grados } \\
\text { de libertad }\end{array}$ & $\begin{array}{c}\text { Media } \\
\text { cuadrática }\end{array}$ & Distribución F & Significancia \\
\hline Regresión & 18.160 & 4 & 4.540 & 9.892 & $0.000(\mathrm{a})$ \\
Residual & 65.170 & 142 & 0.459 & & \\
Total & 83.330 & 146 & & & \\
\hline
\end{tabular}

a Variables predictoras: (Constante), impacto, significado, competencia, autodeterminación.

b Variable dependiente: compromiso organizacional.

La regresión implica la influencia del empowerment en los colaboradores para determinar el nivel de compromiso organizacional; sin embargo, cada dimensión de la variable empowerment aporta un nivel distinto de influencia, siendo la dimensión de significados la que posee un mayor nivel en los resultados de la regresión; 
se percibe que los colaboradores incrementan su compromiso hacia la organización al sentir que las metas u objetivos personales no se contraponen a las metas de la organización, encontrando equilibrio y en ocasiones hasta la identificación del colaborador con respecto de la organización.

En menor grado de influencia se encuentran las dimensiones de autodeterminación e impacto, para tal efecto también se puede predecir que existe compromiso hacia la organización cuando los colaboradores experimentan sensación de independencia para decidir si inician una actividad y cómo desarrollarla dentro del ámbito de sus funciones, o cuando los colaboradores perciben que ellos contribuyen de manera significativa en los resultados para lograr los objetivos organizacionales, pues las dimensiones de autodeterminación e impacto estudian dichas acepciones respectivamente.

Sin embargo, la dimensión de competencia tuvo el menor grado de influencia porque aunque los colaboradores experimentaron la creencia de que poseen las habilidades, capacidades y conocimientos para desarrollar sus labores, el nivel de compromiso hacia la organización no se verá fuertemente incrementado por dicha dimensión.

\section{Análisis de Anova}

En el análisis también se evaluó el impacto de las variables moderadoras utilizadas en el levantamiento, para tal efecto se utilizó el análisis de Anova de las variables: tipo de organización, antigüedad laboral, funciones realizadas, género, edad y escolaridad de los colaboradores. El cuadro 7 muestra los resultados para cada una de las variables del modelo y el impacto de los factores antes descritos. Es posible observar que los factores que mostraron significancia estadística para ambas variables son el tipo de organización y la edad de los colaboradores. De manera especial en el compromiso organizacional se observó que resultan estadísticamente significativos la antigüedad en la empresa, el tipo de puesto que desempeñan los empleados y la escolaridad. 


\section{Cuadro 7}

Análisis Anova

\begin{tabular}{|c|c|c|c|c|c|}
\hline & $\begin{array}{c}\text { Suma } \\
\text { de } \\
\text { cuadrados }\end{array}$ & $\begin{array}{c}\text { Grados } \\
\text { de libertad }\end{array}$ & $\begin{array}{c}\text { Media } \\
\text { cuadrática }\end{array}$ & Distribución F & Significancia \\
\hline \multicolumn{6}{|c|}{ Tipo de organización } \\
\hline Empowerment & 4.690 & 5 & 0.938 & 3.286 & 0.008 \\
\hline Compromiso Org. & 8.798 & 5 & 1.760 & 3.329 & 0.007 \\
\hline \multicolumn{6}{|c|}{ Antigüedad dentro de la organización } \\
\hline Empowerment & 1.701 & 2 & 0.850 & 2.832 & 0.062 \\
\hline Compromiso Org. & 4.639 & 2 & 2.319 & 4.244 & 0.016 \\
\hline \multicolumn{6}{|c|}{ Tipo de puesto } \\
\hline Empowerment & 0.454 & 1 & 0.454 & 1.479 & 0.226 \\
\hline Compromiso Org. & 2.223 & 1 & 2.223 & 3.974 & 0.048 \\
\hline \multicolumn{6}{|c|}{ Género de los colaboradores } \\
\hline Empowerment & 0.171 & 1 & 0.171 & 0.553 & 0.458 \\
\hline Compromiso Org. & 0.046 & 1 & 0.046 & 0.081 & 0.777 \\
\hline \multicolumn{6}{|c|}{ Edad de los colaboradores } \\
\hline Empowerment & 3.800 & 3 & 1.267 & 4.403 & 0.005 \\
\hline Compromiso Org. & 6.098 & 3 & 2.033 & 3.764 & 0.012 \\
\hline \multicolumn{6}{|c|}{ Escolaridad de los colaboradores } \\
\hline Empowerment & 2.750 & 4 & 0.687 & 2.314 & 0.060 \\
\hline Compromiso Org. & 15.581 & 4 & 3.895 & 8.164 & 0.000 \\
\hline
\end{tabular}

\section{Conclusiones y sugerencias}

El estudio permite comprobar que el empowerment es un predictor del compromiso organizacional. Esta conclusión depende de la generalización en el análisis de ambas variables, como ha quedado determinado. Sin embargo, si se evalúa de manera individual la relación de cada una de las cuatro dimensiones de la variable independiente (significado, competencia, autodeterminación e impacto) con respecto a las tres dimensiones de la variable dependiente (compromiso continuo, normativo y afectivo), nuestro análisis permite establecer que las dimensiones del empowerment trascienden únicamente sobre la dimensión de compromiso afectivo. Sin embargo, el significado es la única dimensión que produce influencia en el compromiso normativo, mientras que la dimensión de compromiso continuo no percibe el menor nivel de influencia que pueda generar las dimensiones del $\mathrm{em}$ powerment. Es importante señalar que los resultados se ven afectados por el factor de la edad en los colaboradores, independientemente de la organización en la cual se presente la relación entre las variables de investigación. 
Los propietarios y/o administradores pueden conocer el grado que poseen sus colaboradores en cuanto a las dos variables de estudio para crear estrategias que favorezcan el desarrollo profesional y laboral de sus colaboradores, que fomentan la integración de su personal, así como la adhesión de ellos hacia la organización.

\section{Figura 3}

\section{Modelo ajustado de la influencia del empowerment en el compromiso organizacional}

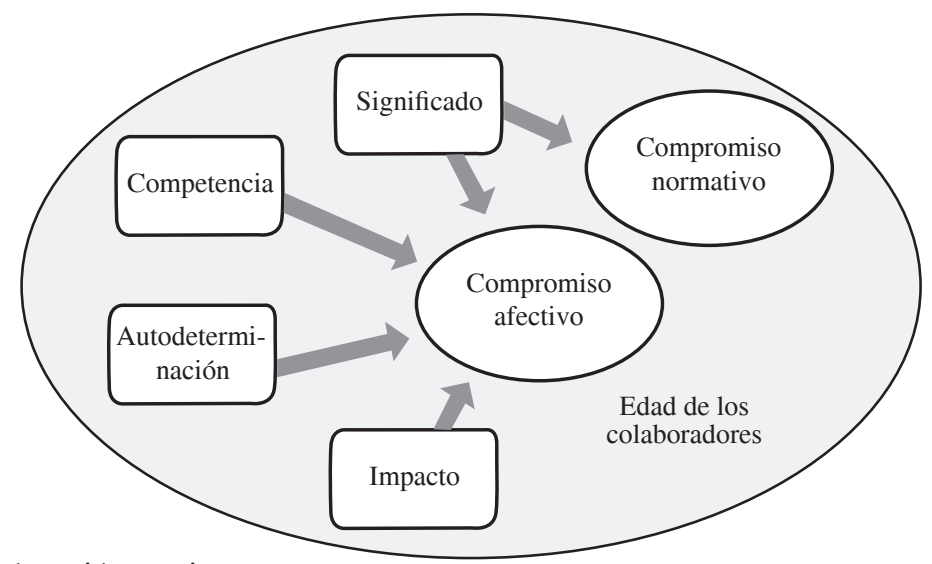

Fuente de elaboración propia

Con respecto a los objetivos planteados en el estudio se establecen las siguientes conclusiones:

- Conocer si existe relación al otorgar empowerment a los colaboradores con respecto al compromiso organizacional. Se determinó rechazar la hipótesis nula y, por consiguiente, aceptar la hipótesis alternativa dado que ha quedado establecido que el empowerment permite predecir al compromiso organizacional.

- Con relación a las hipótesis complementarias para determinar el impacto de cada una de las dimensiones del empowerment organizacional sobre cada una de las dimensiones del compromiso organizacional, se establece que cada una de las cuatro dimensiones del empowerment tiene impacto individual sobre el compromiso organizacional; sin embargo, este impacto sólo es significativo sobre el compromiso afectivo. Adicionalmente, en el caso del significado como dimensión de empowerment tiene relación predictiva sobre el compromiso normativo. La relación de las dimensiones de ambas variables ajustada a los resultados obtenidos se muestra en el cuadro 2. 
Es importante señalar que la obtención de este modelo ajustado permite establecer condiciones para promover el compromiso organizacional de los colaboradores. Para ello, es necesario la implementación de actividades como una comunicación clara y abierta, la implementación de equipos de trabajo, consecuentemente, crear sinergia en los integrantes de los equipos para lograr el cambio de una organización piramidal a una más plana en su estructura, siempre que dichas actividades estén encaminadas a generar en los colaboradores la sensación de que son dueños de las actividades por realizar y que poseen la capacidad para desarrollar satisfactoriamente las funciones que les encomienden, incrementando así el empowerment en ellos, mediante la identificación de metas personales y organizacionales, la capacidad para desarrollar sus funciones, el poder de decisión para definir la mejor forma de realizar sus funciones y percibir los resultados obtenidos con base en el desempeño que ha realizado el colaborador.

\section{Sugerencias}

Los resultados del estudio permiten establecer un marco de referencia significativo respecto con las dos variables estudiadas; sin embargo, sería recomendable ampliar el mismo desde tres consideraciones principales:

- Realizar un estudio más amplio que integre el factor de tamaño de las organizaciones, especialmente en lo referente a las grandes y empresas.

- Incluir aseveraciones relacionadas con base en la teoría de compromisos múltiples.

- Ampliar el diseño muestral para establecer una representación geográfica más importante.

\section{Referencias}

Arciniega, L. (2002). Compromiso organizacional México ¿Cómo hacer que la gente se ponga la camiseta? México: Limusa.

Bayona, C., S. GoÑi y C. MadorRán (1999). "Compromiso organizacional: implicaciones para la gestión estratégica de los recursos humanos". Revista Europea de Dirección y Economía de la Empresa. 139-149. 
Bogler, R. y A. SOMECH (2004). "Influence of teacher empowerment on teachers' organizational commitment, professional commitment and organizational citizenship behavior in schools". Teaching and Teacher Education, Vol. 20, 277-289.

Chen, H-F (2008). "The Impacts of Empowerment on Organizational Commitment under a Changing Environment - Examining the Moderating Effects of Locus of Control and Perceived Psychological Contract Breach". Department of International Business, Soochow University, Vol. 18, No. 2, 1-26.

Davis, K. y Nwestrom, J. (2000). Comportamiento humano en el trabajo. México: Mc. Graw Hill.

Dee, J., A. B. Henkin y L. Duemer (2003). "Structural antecedents and psychological correlates of teacher empowerment". Journal of Educational Administration, Vol. 4, No. 1, 257-277.

Gómez, D. G. (2006). K Sigma: Control de procesos para mejorar la calidad de la enseñanza (cómo identificar entre lo mucho que es trivial lo poco que es crítico). México: WK Educación.

JAFFe, D. T. y C. D. ScotT (2007). Empowerment. México: Oxford University Press.

JANSSEN, O. (2004). "The barrier effect of conflict with superiors in the relationship between employee empowerment and organizational commitment". Work \& Stress, Vol. 18, No. 1, 56-65.

KRISHNA, Y. R. (2006). "Psychological empowerment and organizational commitment: An empirical study of software programmers in India", Hyderabad Andhra Pradesh, India, 1-16.

Kuokkanen, L., H. Leino-Kilpi y J. Katajisto (2003). "Nurse Empowerment, Job-Related Satisfaction, and Organizational Commitment". Journal of Nursing Care Quality, Vol. 18, No. 3, 184-192.

Malhotra, N. K. (2004). Investigación de mercados. $4^{\text {a }}$ ed., México: Pearson Educación. 
Meyer, J. P. y N. J. Allen (1997). Commitment in the Workplace. Theory, research and application. Thousand Oaks, California: Sage publications, inc.

Molina, R. (2006). Empowerment ¿actitud mental?, ¿técnica?, ¿estrategia? o ¿moda? México: Trillas.

Mowday, R. y R. SteErs (1979). Employee-Organization Linkages. New York: Academic press.

Nunnally, J. C. (1978). Psychometric theory. New York: Mc. Graw Hill.

Rego, A. y Souto, S. (2004). "La relación entre los climas organizacionales autentizóticos y las variables intención de salida de la organización, el compromiso organizacional y el estrés". Revista Latinoamericana de Administración, Universidad de los Andes, Bogotá, Colombia, No. 32, 77-102.

Robbins, S. P. (2004). Comportamiento organizacional $10^{a}$ ed., México: Prentice Hall.

SAlKind, N. J. (1999). "Métodos de Investigación”. 3. ed., México: Prentice Hall. Tercera edición.

Spreitzer, G. M. (1995). "Psychological Empowerment in the Workplace: Dimensions, Measurement, and Validation”. The Academy of Management Journal, Vol. 38, No. 5, 1442-1465. 